\title{
A Fair QoS-Based Resource Allocation Scheme for a Time-Slotted Optical OV-CDMA Network: A Unified Approach
}

\author{
Elie Inaty, Member, IEEE, Robert Raad, Paul Fortier, Senior Member, IEEE, and
} Hossam M. H. Shalaby, Senior Member, IEEE

\begin{abstract}
In this paper, we develop a fair resource allocation scheme for a multiclass time-slotted optical overlapped code-division multiple-access (OV-CDMA) network. The resource management scheme is fair in the sense that the users have their power and rate allotted according to their quality of service $(\mathrm{Q} O S)$ requirements. In addition, no class of users can dominate over the other classes. A unified framework for the resource controlling scheme is proposed. It consists of simply finding a single control parameter based on which the optimal transmission rate and power are obtained for every class of users. Analytical results show that the maximum transmission rate for a given class of users is only achievable when the users in that class are transmitting with the highest possible laser transmission power. In addition, we have demonstrated that the optimal transmission rates can be obtained via the solution of the rate characteristic polynomial. It is shown that our proposed scheme provides a substantial improvement in the system capacity while preserving the required fairness criteria.
\end{abstract}

Index Terms-Capacity, multiclass, multirate, optical overlapped code-division multiple-access (OV-CDMA), overlapping coefficient, power control, rate control.

\section{INTRODUCTION}

$\mathbf{M}$ ANY optical networks have been deployed with the aim of accommodating novel information-services generated by modern communication terminals like high-speed personal computers, FTTX networks and many others [1]. Those services require a huge amount of bandwidth, multirate transmission capabilities, and consume more power due to the amount of data that can be handled. In this regard, we believe that this paper can contribute to this research trend.

The optical overlapped code-division multiple-access (OVCDMA) system has been proposed in [2] to increase the transmission rate of an optical communication system by overlapping consecutive bits while transmitted along the fiber. The question that arises here is: what extent of bit overlapping and hence

Manuscript received February 5, 2008; revised June 17, 2008. Current version published February 13, 2009.

E. Inaty is with the Department of Computer Engineering, University of Balamand, El-Koura, Lebanon (e-mail: elie.inaty @ balamand.edu.lb).

R. Raad and P. Fortier are with the Department of Electrical and Computer Engineering, Laval University, Quebec, QC G1K 7P4, Canada (e-mail: robert.j. raad@balamand.edu.lb; fortier@gel.ulaval.ca).

H. M. H. Shalaby is with the Department of Electrical Engineering, University of Alexandria, Alexandria 21544, Egypt (e-mail: shalaby@ieee.org).

Color versions of one or more of the figures in this paper are available online at http://ieeexplore.ieee.org.

Digital Object Identifier 10.1109/JLT.2008.929123 of transmission rate can the system admit such that the signal can be successfully received? In fact, all depends on the interference level and on the signal power that is injected into the fiber, or in other words on the signal-to-interference ratio (SIR). For this reason, we consider the SIR as the quality of service (QoS) metric in our analysis. Resource allocation schemes are necessary to enhance the performance of optical networks in order to guarantee successful data transmission and to satisfy the users' requirements. Many algorithms are found in the literature to cope with this problem. However, most of them have been proposed to deal with wireless systems [3]-[6], while few have been investigated to deal with optical-channel-based networks [7]-[12].

For instance, a nonlinear programming power control algorithm has been proposed in [7] to maximize the capacity of multirate optical fast frequency hopping code division multiple access (OFFH-CDMA) systems constrained by a predefined QoS. In [10], a power control algorithm, based on optical power selector has been inspected for a multirate optical DS-CDMA system. In addition, an adaptive overlapped pulse-position modulator, employed to create multirate and multiquality transmission schemes, has been investigated in [11] for optical CDMA (OCDMA) networks. Moreover, the power control problem is also addressed in [12] for temporal prime coded OCDMA system. Lately, we have proposed a novel hybrid power/rate control algorithm for the optical OV-CDMA system [2]. The problem of this strategy is that it is not fair in the sense that there is at most one class of users that has an overlapping coefficient between zero and full overlap, and the remaining classes either transmit with full overlap or with no overlap.

The first contribution of this paper is to propose a unified framework for allocating and controlling the transmission rate and power in a way that it can be applied for any expression of the system capacity. In addition, it is generalized to a timeslotted packetized system [8]. The second contribution is the reduction of the optimization search space into one dimensional space. This target guaranties the simplicity of the algorithm, which is an important requirement in optical networks. Our third contribution is to solve the problem of unfairness in the resource allocation strategy presented in [7].

The rest of the work is organized as follows. In Section II, we present the system model where we introduce the main system parameters and the network under study. The performance of the network at the physical layer has been discussed in Section III. In Section IV, we investigate the proposed resource allocating 


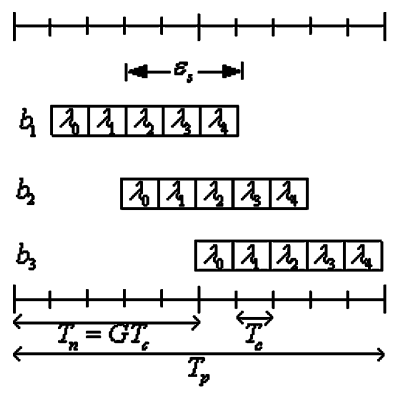

a)

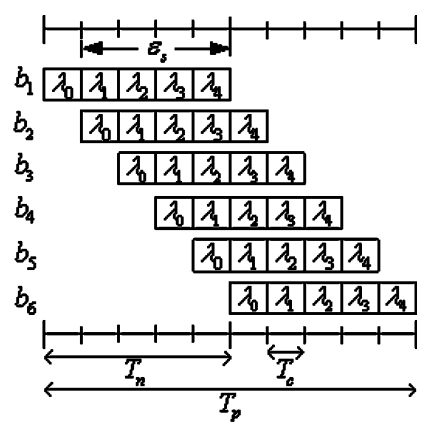

b)
Fig. 1. An optical OV-CDMA packet model for a single user in a given time slot. (a) $\varepsilon_{s}=3$. (b) $\varepsilon_{s}=4$.

and controlling scheme of the overlapped CDMA-based packet network. In Section V, we define and we analyze the network capacity upon which we determine the optimal control parameter that optimizes the allocated resources among classes. Simulation results and discussion are provided in Section VI. Finally, we close our work with a conclusion in Section VII.

\section{SYSTEM MODEL}

Consider a multiclass time-slotted OV-CDMA system in an optical packet network in which $K$ users are connected in a star topology as the one proposed in [8]. The nominal bit duration is $T_{n}$ and its corresponding nominal rate is $R_{n}=1 / T_{n}=1 / G T_{c}$, where $G$ is the processing gain (PG) and $T_{c}$ is the chip interval. Due to the linearity of the encoder-decoder set, multibits will be coded and transmitted when the data rate increases beyond $R_{n}$. This introduces a bit overlapping coefficient $\varepsilon_{s}$, which represents the number of overlapping chips between two consecutive bits as revealed in Fig. 1.

The users are assigned different optical code signatures of equal PG and are distributed over $S$ classes. Each class $j, \forall j \in$ $\{0,1, \ldots, S-1\}$, is characterized by its own and unique QoS, so that it has its proper transmission power $P_{j}$ and overlapping coefficient $\varepsilon_{j}$. The users transmit their coded bits in a given time slot in the form of packets of nominal length of $L$ bits. However, due to the overlapping process, the maximum number of bits which a user can transmit per packet exceeds $L$ as presented in [8] and shown in Fig. 1. This figure illustrates an example of the overlapping process in a packet time slot. In this example, the packet length is $L=2$, and the PG is $G=5$. For $\varepsilon_{s}=0$, the transmission rate is the nominal rate, which is equal to two bits per packet. When the overlapped coefficient is increased to $\varepsilon_{s}=3$ as shown in Fig. 1(a), the transmission rate is increased to three bits per packet. On the other hand, Fig. 1(b) shows the case where $\varepsilon_{s}=4$. Accordingly, the transmission rate is six bits per packet. Note that $\lambda_{i}$ represents the assigned wavelength in the $i$ th time instance.

Let $0 \leq \varepsilon_{j} \leq G-1, \forall j \in\{0,1, \ldots, S-1\}$, be the overlapping coefficient and $X_{b, j}$ to be the total number of overlapped bits in a packet time slot. The expression of $X_{b, j}$ can be found in [8] and it is given by

$$
X_{b, j}=\left\lfloor\frac{L G-\varepsilon_{j}}{G-\varepsilon_{j}}\right\rfloor .
$$

Consequently, the achievable transmission rate of class- $j$ users is given by

$$
R_{j}=\frac{X_{b, j}}{L} R_{n}
$$

\section{SySTEM PERFORMANCE}

We are interested in evaluating the average SIR per bit in a given time slot for every class- $j$, which has its own power and overlapping coefficient and supporting $K_{j}$ users. Then, the average SIR, as derived in [8], is given by

$$
\frac{\operatorname{SIR}_{j}(\varepsilon, \mathbf{P})=}{\left(K_{j}-1\right) P_{j} \bar{\sigma}_{I_{k_{j}}}^{2}\left(\varepsilon_{j}\right)+\sum_{\substack{s=0 \\ s \neq j}}^{S-1} \sum_{\substack{s=1 \\ s \neq j}}^{K_{s}} P_{s} \bar{\sigma}_{I_{k_{s}}}^{2}\left(\varepsilon_{s}\right)+\sigma_{n}^{2}}
$$

where $\varepsilon$ and $\mathbf{P}$ are vectors of the overlapping coefficients and the transmission power of all classes, respectively. $\bar{\sigma}_{I_{k j}}^{2}$ is the average multiple access interference (MAI) power imposed by a class- $j$ user on the desired bit in a given time slot, and it is computed as [8] shown in (4) at the bottom of the page, and see (5) at the bottom of the next page, where $X_{r, j}=\left\lceil\varepsilon_{j} /\left(G-\varepsilon_{j}\right)\right\rceil$ as computed in [1]. In addition, $H_{\nu}^{2}\left(0, q_{\nu}^{(i)}\right)$ and $H_{\nu}^{2}\left(q_{\nu}^{(i)}, G\right)$ are the partial period Hamming correlation functions [1], [2]. $\sigma_{n}^{2}$ represents the power of the other sources of noise modeled as Gaussian noise. It involves thermal noise, shot noise, dark current, and surface leakage current [13], [14].

$$
\begin{aligned}
\bar{\sigma}_{I_{k_{j}}}^{2}= & 2 \sum_{i=0}^{X_{b, j}-X_{r, j}-1}\left[\sum_{\nu=-i}^{-1} H_{\nu}^{2}\left(0, q_{\nu}^{(i)}\right)+\sum_{\nu=0}^{X_{r, j}} H_{\nu}^{2}\left(q_{\nu}^{(i)}, G\right)\right] \frac{\left(X_{b, j}-X_{r, j}\right)}{X_{b, j}} \\
& +\sum_{i=X_{b, j}-X_{r, j}}^{X_{r, j}-1}\left[\sum_{\nu=-i}^{-1} H_{\nu}^{2}\left(0, q_{\nu}^{(i)}\right)+\sum_{\nu=0}^{X_{b, j}-i-1} H_{\nu}^{2}\left(q_{\nu}^{(i)}, G\right)\right] \frac{\left(2 X_{r, j}-X_{b, j}\right)}{X_{b, j}} \\
X_{b, j}-2 X_{r, j} \leq 0 &
\end{aligned}
$$


Assuming one-coincidence sequences, the average value of the partial period Hamming correlation functions can be easily computed and they are given by

$$
\begin{aligned}
\bar{H}_{\nu}^{2}\left(0, q_{\nu}^{(i)}\right) & =\frac{\left(G+\nu\left(G-\varepsilon_{j}\right)\right)}{F} \\
\bar{H}_{\nu}^{2}\left(q_{\nu}^{(i)}, G\right) & =\frac{\left(G-\nu\left(G-\varepsilon_{j}\right)\right)}{F}
\end{aligned}
$$

where $F$ is total number of available wavelengths from which the code is constructed and $\nu$ is the bit index in the packet. Using (6) and (7) in (4) and (5), we get $\bar{\sigma}_{I_{k}}^{2}$ in terms of the number of bits per packet as follows in (8) at the bottom of the page, where $A_{i}$ for $0 \leq i \leq 4$ and $B_{j}$ for $0 \leq j \leq 3$ are constant coefficients, functions of the OV-CDMA system parameters $G$, $F$, and $L$. Their values are shown in Appendix.

We consider that the users are dispersed among classes according to a given distribution with a probability mass function $(\mathrm{PMF}) p^{(j)}$ such that $\sum_{j=0}^{S-1} p^{(j)}=1$. Therefore, the total number of users in class- $j$ is given by $K_{j}=K p^{(j)}$ for $0 \leq j \leq$ $S-1$.

\section{Resource Allocation Strategy}

Our strategy is based of the assumption that the QoS of class- $j$ in the presence of other classes, given in (3), should meet the requirement

$$
S I R_{j} \geq \beta_{j}
$$

We define the solitary class- $j$ SIR as the desired signal power over the power of a class- $j$ interferer. It is given by

$$
\gamma_{j}=\frac{G^{2}}{\bar{\sigma}_{I_{k j}}^{2}}
$$

In addition, we define the nominal signal-to-noise ratio (SNR) that is common to all classes as

$$
\gamma_{n}=\frac{G^{2}}{\sigma_{n}^{2}}
$$

Using (10) and (11), the minimum requirements in (9) (which means with equality) gives $S$ linear equations in the laser transmission power of each class as follows:

$$
\sum_{s=0}^{S-1} \frac{K_{s}}{\gamma_{s}} P_{s}-\left(\frac{1}{\gamma_{j}}+\frac{1}{\beta_{j}}\right) P_{j}+\frac{1}{\gamma_{n}}=0, \quad \forall 0 \leq j \leq S-1 .
$$

The solution of (12) represents the power allocation for all classes. Let $\Gamma=\operatorname{diag}\left(1 / \gamma_{0}, \ldots, 1 / \gamma_{S-1}\right), B=$ $\operatorname{diag}\left(1 / \beta_{0}, \ldots, 1 / \beta_{S-1}\right)$, and $\mathbf{K}=\operatorname{diag}\left(K_{0}, \ldots, K_{S-1}\right)$ be $S \times S$ diagonal matrices. $\mathbf{P}=\left(P_{0}, \ldots, P_{S-1}\right)^{T}$ is the vector of the unknown transmission powers of the $S$ classes, and $\mathbf{e}_{S \times 1}$ is a unity vector. Also, let $\mathbf{N}_{D}=\Gamma+B$, $\mathbf{M}_{D}=\left(\mathbf{N}_{D}-B\right) \mathbf{K}=\Gamma \mathbf{K}$. Then, the linear system in (12) can be reduced to $(-M) \mathbf{P}=1 / \gamma_{n} \mathbf{e}$, where $M^{T}=\mathbf{M}_{D} \mathbf{e e}^{T}-\mathbf{N}_{D}$ is the system-performance matrix. Thereby, the power vector is given by

$$
\mathbf{P}=\frac{1}{\gamma_{n}}\left(-M^{-1}\right) \mathbf{e}
$$

The objective here is to determine the optical power that maintains the demanded QoS while forcing the chip overlapping to occur to a certain extent that will be determined later in order to increase the transmission rate of the users. From linear algebra, we have $\left(-M^{-1}\right)^{T}=\left(-M^{T}\right)^{-1}$. Applying the Sherman-Morrison matrix inversion formula [15], which states that for any nonsingular matrix $A \in \mathbb{R}^{n \times n}, b, c \in \mathbb{R}^{n}$ and $1+c^{T} A^{-1} b \neq 0$

$$
\left(A+b c^{T}\right)^{-1}=A^{-1}-\frac{A^{-1} b c^{T} A^{-1}}{1+c^{T} A^{-1} b}
$$

we obtain

$$
\left(\mathbf{N}_{D}-\mathbf{M}_{D} \mathbf{e} \mathbf{e}^{T}\right)^{-1}=\left(\mathbf{I}+\frac{1}{\zeta} \mathbf{N}_{D}^{-1} \mathbf{M}_{D} \mathbf{e e}^{T}\right) \mathbf{N}_{D}^{-1}
$$

$$
\begin{aligned}
\bar{\sigma}_{I_{k_{j}}}^{2}= & 2 \sum_{i=0}^{X_{r, j}-1}\left[\sum_{\nu=-i}^{-1} H_{\nu}^{2}\left(0, q_{\nu}^{(i)}\right)+\sum_{\nu=0}^{X_{r, j}} H_{\nu}^{2}\left(q_{\nu}^{(i)}, G\right)\right] \frac{X_{r, j}}{X_{b, j}} \\
& +\sum_{i=X_{r, j}}^{X_{b, j}-X_{r, j}-1}\left[\sum_{\nu=-X_{r, j}}^{-1} H_{\nu}^{2}\left(0, q_{\nu}^{(i)}\right)+\sum_{\nu=0}^{X_{r, j}} H_{\nu}^{2}\left(q_{\nu}^{(i)}, G\right)\right] \frac{\left(X_{b, j}-2 X_{r, j}\right)}{X_{b, j}} \\
X_{b, j}-2 X_{r, j}>0 &
\end{aligned}
$$

$$
\bar{\sigma}_{I_{k_{j}}}^{2}= \begin{cases}\frac{-\frac{1}{3}\left(A_{4} X_{b, j}^{4}+A_{3} X_{b, j}^{3}+A_{2} X_{b, j}^{2}+A_{1} X_{b, j}+A_{0}\right)}{F(L-1)^{3}\left(X_{b, j}-1\right) X_{b, j}} & L \leq \frac{3 X_{b, j}}{X_{b, j}+2} \\ \frac{\left(B_{3} X_{b, j}^{3}+B_{2} X_{b, j}^{2}+B_{1} X_{b, j}+B_{0}\right)}{F(L-1)^{3} X_{b, j}}, & L>\frac{3 X_{b, j}}{X_{b, j}+2}\end{cases}
$$


where $\mathbf{I}$ is an $\times$ identity matrix. This implies (16) at the bottom of the page, where

$$
\zeta \equiv \sum_{s=0}^{S-1}\left[\frac{K_{s}}{1+\frac{\beta_{s}}{\gamma_{s}}}\right]-(K-1), \quad \zeta>0 .
$$

Consequently, substituting (15) in (13) we get

$$
\mathbf{P}=\frac{1}{\gamma_{n}}\left(\mathbf{e}^{T}\left(\mathbf{I}+\frac{1}{\zeta} \mathbf{N}_{D}^{-1} \mathbf{M}_{D} \mathbf{e e}^{T}\right) \mathbf{N}_{D}^{-1}\right)^{T}=\frac{\mathbf{N}_{D}^{-1} \mathbf{e}}{\zeta \gamma_{n}}
$$

When $\zeta=0$, the optical intensity is extremely high, which leads to nonlinear effects and causes severe degradation of the signal [14]. To avoid this situation, we consider that the optical power is upper bounded by $\Pi=\left(\pi_{0}, \ldots, \pi_{S-1}\right)^{T}$ such that

$$
P_{j} \leq \pi_{j}, \quad \forall 0 \leq j \leq S-1
$$

Lemma 1: The linear system in (12) constrained by (19) has a solution if and only if the solitary class- $j$ SIR, $\gamma_{j}$, is lower bounded by

$$
\gamma_{j}^{*}=\frac{(K-1) \pi_{j}}{\sum_{s=0}^{S-1} \frac{K_{s} \pi_{s}}{\beta_{s}}-(K-1) \frac{\pi_{j}}{\beta_{j}}-\frac{1}{\gamma_{n}}} .
$$

Proof: The power allocation vector can be written as

$$
\mathbf{P}=\frac{\mathbf{N}_{D}^{-1} \mathbf{e}}{\gamma_{n}\left((\mathbf{K e})^{T} B \mathbf{N}_{D}^{-1} \mathbf{e}-\mathbf{e}^{T} \mathbf{K}^{T} \mathbf{e}+1\right)} \leq \Pi .
$$

By arranging the terms we obtain

$$
\mathbf{N}_{D}^{-1} \mathbf{e} \geq \gamma_{n}(K-1)\left(-\left(\mathbf{I}-\gamma_{n} \Pi(\mathbf{K e})^{T} B\right)^{-1}\right) \Pi .
$$

Using Sherman-Morrison formula [15] to compute the inverse, we get

$$
\left(\mathbf{I}-\gamma_{n} \Pi(\mathbf{K e})^{T} B\right)^{-1}=\mathbf{I}+\frac{\gamma_{n} \Pi(\mathbf{K e})^{T} B}{1-\gamma_{n} \Pi^{T} B^{T}(\mathbf{K e})} .
$$

This yields

$$
\mathbf{N}_{D}^{-1} \mathbf{e} \geq \frac{\gamma_{n}(K-1)}{\gamma_{n}(\mathbf{K e})^{T} B \Pi-1} \Pi
$$

Then, from (22) we evaluate the upper bound of $\gamma_{j}$ for each class. This gives

$$
\left(\frac{\beta_{0}}{1+\frac{\beta_{0}}{\gamma_{0}}}, \ldots, \frac{\beta_{S-1}}{1+\frac{\beta_{S-1}}{\gamma_{S-1}}}\right)^{T} \geq \frac{\gamma_{n}(K-1)}{\gamma_{n}(\mathbf{K e})^{T} B \Pi-1} \Pi .
$$

Thus, we can write

$$
\frac{1}{\gamma_{j}} \leq \frac{\sum_{s=0}^{S-1} \frac{K_{s} \pi_{s}}{\beta_{s}}-\frac{1}{\gamma_{n}}}{(K-1) \pi_{j}}-\frac{1}{\beta_{j}}
$$

which implies that the SIR of solitary class- $j$ should satisfy

$$
\gamma_{j} \geq \gamma_{j}^{*} \equiv \frac{(K-1) \pi_{j}}{\sum_{s=0}^{S-1} \frac{K_{s} \pi_{s}}{\beta_{s}}-(K-1) \frac{\pi_{j}}{\beta_{j}}-\frac{1}{\gamma_{n}}} .
$$

Which completes the proof of the lemma.

Lemma 2: For a given value of $\pi_{j}$ and $\beta_{j}, \gamma_{j}^{*}$ in (20) always exists $\forall 0 \leq j \leq S-1$.

Proof: We know that $\gamma_{j}^{*}>0$ which implies that

$$
\sum_{s=0}^{S-1} \frac{K_{s} \pi_{s}}{\beta_{s}}-(K-1) \frac{\pi_{j}}{\beta_{j}}-\frac{1}{\gamma_{n}}>0, \quad \forall 0 \leq j \leq S-1 .
$$

By averaging (24) over all classes, we obtain

$$
E_{s}\left\{\frac{\pi_{s}}{\beta_{s}}\right\}>\frac{1}{\gamma_{n}}
$$

On the other hand, from (24) we have

$$
\frac{\pi_{j}}{\beta_{j}}<\frac{K E_{j}\left\{\frac{\pi_{j}}{\beta_{j}}\right\}-\frac{1}{\gamma_{n}}}{K-1}, \quad \forall j \in\{0,1, \ldots, S-1\} .
$$

In addition, (24) can be written in a matrix form as

$$
\left(\mathbf{K e e}^{T}-(K-1) \mathbf{I}\right)^{T} B \Pi>\frac{1}{\gamma_{n}} \mathbf{e} .
$$

Then, by solving the linear system (27) for $\pi_{j} / \beta_{j}$, we get

$$
\begin{aligned}
& B \Pi>\frac{1}{\gamma_{n}}\left(-\left((K-1) \mathbf{I}-\mathbf{K e e}^{T}\right)^{-1}\right)^{T} \mathbf{e} \\
\Rightarrow & \frac{\pi_{j}}{\beta_{j}}>\frac{1}{\gamma_{n}}, \quad \forall j .
\end{aligned}
$$

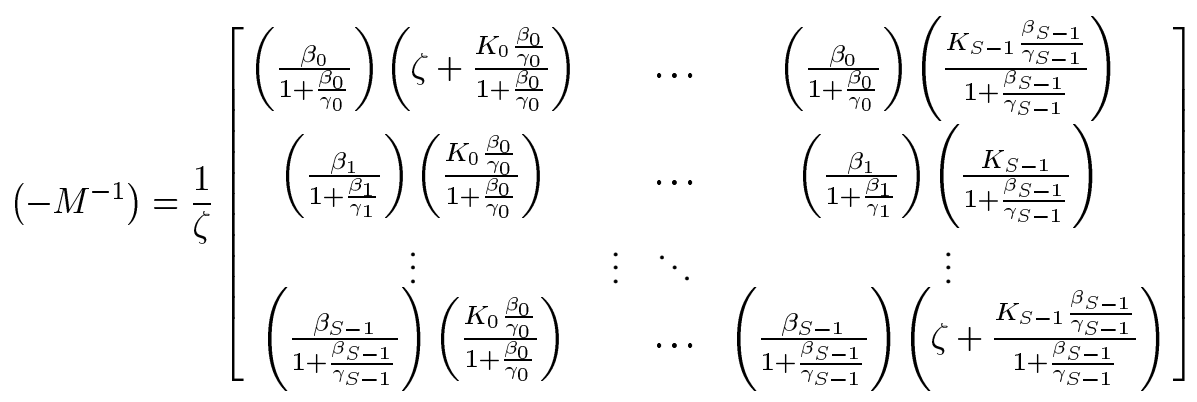


Thus, we can write

$$
\frac{1}{\gamma_{n}}<\frac{\pi_{j}}{\beta_{j}}<\frac{K E_{j}\left\{\frac{\pi_{j}}{\beta_{j}}\right\}-\frac{1}{\gamma_{n}}}{K-1}, \quad \forall 0 \leq j \leq S-1 .
$$

Using (30), it is clear that $\gamma_{j}^{*}$ is bounded as follows:

$$
0<\gamma_{j}^{*}<\infty \quad \forall 0 \leq j \leq S-1 .
$$

This means that $\gamma_{j}^{*}$ is finite, therefore it always exits.

Notice that (30) yields the admissible interval of the power convergence.

In this work, the power allocation is assumed to be fair among all classes, which means that no class can dominate the others due to a high power allocation. Hence, let the ratio $\pi_{j} / \beta_{j}$ be arbitrarily identical for all classes. Thus, let $\alpha$ be a control parameter whose role is to manage the power level that is necessary to satisfy the required QoS while maintaining the fairness criterion. It is given by

$$
\frac{\pi_{j}}{\beta_{j}}=\frac{\alpha}{\gamma_{n}}, \quad \forall 0 \leq j \leq S-1 .
$$

The domain of $\alpha$ can be obtained from Lemma 2. The optimal value of $\alpha$ should be the one that maximizes the network capacity. On the other hand, substituting (32) in (23) yields

$$
\gamma_{j}^{*}=\frac{\alpha}{\alpha-1}(K-1) \beta_{j} .
$$

\section{A. Rate Allocation and Control Scheme}

Note that, as $\gamma_{j}$ increases, $\bar{\sigma}_{I_{k_{j}}}^{2}$ decreases. This, in turn, results in a reduction of the number of overlapping bits per packet, and hence a reduction of the transmission rate. From (10) and (23), we define the rate characteristic polynomial as the difference between the average interference variance per bit period and the target interference variance. It is given by

$$
\rho\left(R_{j}\right) \equiv \bar{\sigma}_{I_{k_{j}}}^{2}-\frac{G^{2}}{\gamma_{j}^{*}} .
$$

It is clear that because $\gamma_{j} \geq \gamma_{j}^{*}, \rho\left(R_{j}\right)$ should be less or equal to zero. By setting $\rho\left(R_{j}\right)=0$, we compel the system to hit the target MAI. Since $\bar{\sigma}_{I_{k_{j}}}^{2}$ is a function of $X_{b, j}$, and hence of $R_{j}$, the rate-characteristic polynomial permit us to determine the feasible transmission rate region as well as to extract the transmission rate that meets the QoS requirement. Therefore, the admissible region of the transmission rate is given by

$$
\mathfrak{R}=\left\{\begin{array}{l}
R_{j}: R_{n} \leq R_{j} \leq \frac{(L-1) G+1}{L} R_{n}, \text { s.t } \rho\left(R_{j}\right) \leq 0, \\
\forall j \in\{0,1, \ldots, S-1\}
\end{array}\right\} .
$$

Lemma 3: Let $\beta^{(\ell)}$ and $\beta^{(u)}$ be the lower and the upperbound QoS, respectively. They are given by

$$
\beta^{(\ell)}=\frac{G^{2}[1-(1 / \alpha)]}{(K-1) \max \left\{\bar{\sigma}_{I_{k_{j}}}^{2}\right\}}, \beta^{(u)}=\frac{G^{2}[1-(1 / \alpha)]}{(K-1) \min \left\{\bar{\sigma}_{I_{k_{j}}}^{2}\right\}} .
$$

The maximum admissible transmission rate of class $-j$ users is given by the highest admissible root of the rate-characteristic polynomial when $\beta^{(\ell)} \leq \beta_{j} \leq \beta^{(u)}$, and it is $R^{(u)}$ when $\beta_{j}<\beta^{(\ell)}$. The system has no feasible solution when $\beta_{j}>\beta^{(u)}$

Proof: Let $R^{(\ell)}=R_{n}$ and $R^{(u)}=$ $[((L-1) G+1) / L] R_{n}$ be the lower and the upper possible transmission rates of the system, respectively. Also, let $R_{j} \in \mathfrak{R}$ be the rate for class- $j$ users. In addition, we define $r_{j}^{(\kappa)}$ as the $\kappa$ th root of the rate-characteristic polynomial $\rho\left(R_{j}\right)=0$ of class $-j$. From (8), we note that $\rho\left(R_{j}\right)=0$ can be either a third- or a fourth-order polynomial. Thus, $\kappa \in \mathbf{I}_{r} \equiv\{1,2,3,4\}$. On the other hand, $\rho\left(R_{j}\right)=0$ can have either one, two or three extremes. Thus, we define $R_{j}\left(\rho_{\min }\right)$ and $R_{j}\left(\rho_{\max }\right)$ to be the transmission rates for class- $j$ users that correspond to any minimum or any maximum of $\rho$, respectively.

Given a class- $j$ whose users transmit at $R^{(u)}$, then the induced MAI is the maximum possible one. Hence, let $\bar{\sigma}_{I_{k_{j}}}^{2}\left(R^{(u)}\right) \equiv$ $\bar{\sigma}_{\max }^{2}$. This yields $\bar{\sigma}_{I_{k_{j}}}^{2}\left(R_{j} \leq R^{(u)}\right) \leq \bar{\sigma}_{\max }^{2}$, so $\gamma_{j} \geq \gamma^{(\ell)} \equiv$ $G^{2} / \bar{\sigma}_{\max }^{2}$. On the other hand, $\forall \kappa, r_{j}^{(\kappa)}$ corresponds to the case where $\rho\left(R_{j}=r_{j}^{(\kappa)}\right)=0$, i.e., $\gamma_{j}=\gamma_{j}^{*}$. By hypothesis, this implies that $r_{j}^{(\kappa)}$ satisfy the QoS requirement (i.e., $S I R_{j} \geq \beta_{j}$ ); hence, $\gamma^{(\ell)} \leq \gamma_{j}^{*}$. Using (33), the lowest possible QoS allowed for all classes is given as

$$
\beta^{(\ell)}=\frac{G^{2}[1-(1 / \alpha)]}{(K-1) \bar{\sigma}_{\max }^{2}} .
$$

This means that the minimal solitary class- $j$ SIR cannot go beneath the one that is determined by the system as long as $\beta_{j} \geq \beta^{(\ell)}$. Thus, the polynomial roots are always smaller than the upper bound transmission rate.

Notice that as the transmission rate $R_{j}$ moves toward $R^{(\ell)}$, $\gamma_{j}$ goes far beyond $\gamma_{j}^{*}$ and Lemma 1 is satisfied, which in turn yields

$$
\gamma_{j}^{*} \leq \frac{G^{2}}{\bar{\sigma}_{\min }^{2}} \equiv \gamma^{(u)}
$$

where $\bar{\sigma}_{\min }^{2} \equiv \bar{\sigma}_{I_{k_{j}}}^{2}\left(R^{(\ell)}\right)<\bar{\sigma}_{I_{k_{j}}}^{2}\left(r_{j}^{(\kappa)}\right)$.

Combining (33) with (37), we obtain the maximum QoS for all classes below which $r_{j}^{(\kappa)} \geq R^{(\ell)}$ as

$$
\beta^{(u)}=\frac{G^{2}[1-(1 / \alpha)]}{(K-1) \bar{\sigma}_{\min }^{2}} .
$$

Since $\bar{\sigma}_{\min }^{2}$ is the minimum interference when no overlapping occurs, the maximum number of bits transmitted per time slot is $X_{b, j}=L$ which satisfies the second term in (8). Then, $\bar{\sigma}_{\min }^{2}=$ $L G / F$ and the upper bound QoS becomes

$$
\beta^{(u)}=\frac{G F[1-(1 / \alpha)]}{(K-1) L} .
$$

In order for the rate-characteristic polynomial to have at least one solution $r_{j}^{(\kappa)}$ such that $R^{(\ell)} \leq r_{j}^{(\kappa)} \leq R^{(u)}$, the condition $\beta^{(\ell)} \leq \beta_{j} \leq \beta^{(u)}$ must be fulfilled.

Accordingly, we will determine the admissible region of the transmission rate of all classes by considering the following cases: 
(i) If $R^{(\ell)} \leq r_{j}^{(\kappa)}<R_{j}<R_{j}\left(\rho_{\min }\right) \leq R^{(u)}, \rho\left(R_{j}\right)<0$, and $d \rho / d R_{j}<0$. This means that $\rho\left(R_{j}\right)$ is a monotonically decreasing function of $R_{j}$. In other words, the induced interference diminishes as the transmission rate increases, which is a contradiction. It implies that this region is not feasible in $\mathfrak{R}$.

(ii) If $R^{(\ell)} \leq R_{j}\left(\rho_{\min }\right) \leq R_{j}<r_{j}^{(\kappa)} \leq R^{(u)}, \rho\left(R_{j}\right)<0$ and $d \rho / d R_{j}>0$. In this case, $\rho\left(R_{j}\right)$ is a monotonically increasing function of $R_{j}$. This implies that $\rho\left(R_{j}\right)$ increases toward zero as $R_{j}$ increases toward $r_{j}^{(\kappa)}$, a deduction which agrees with our assumption. Hence, this region is feasible in $\mathfrak{R}$.

(iii) If $R^{(\ell)} \leq R_{j}=r_{j}^{(\kappa)} \leq R^{(u)}$, it is the region of $R_{j}$ that corresponds to the set of roots $r_{j}^{(\kappa)}, \kappa \in \mathbf{I}_{r}$, satisfying $\rho\left(R_{j}\right)=0$. It represents the case where the induced interference is the highest. Thus, this region is also feasible in $\mathfrak{R}$.

As a result, the admissible region can be written as $(E 1 \cup E 2)$ where we have (40) at the bottom of the page.

Now, let the transmission rate $R_{j}^{*} \in E 1 \cup E 2$ represent the highest transmission rate that a user in a given class- $j$ can reach. If $\exists r_{j}, r_{j}=\max _{r_{j}^{(\kappa)} \in \mathfrak{R} \cap(E 1 \cup E 2)}\left(r_{j}^{(\kappa)}\right)$, then

$$
R_{j}^{*}=\sup _{R_{j} \in E 1 \cup E 2}\left(R_{j}\right)=r_{j}
$$

In the case where $\beta_{j}<\beta^{(\ell)}$ or $\beta_{j}>\beta^{(u)}$, the roots of the rate-characteristic equation fall outside the feasible range and we have two situations. First, if $\forall \kappa, r_{j}^{(\kappa)}<R^{(\ell)}, \beta_{j}>\beta^{(u)}$, the system has no feasible solution. Second, if $\forall \kappa, r_{j}^{(\kappa)} \notin \mathfrak{R}$, there exists at least one root $r_{j}^{\left(\kappa^{\prime}\right)}>R^{(u)}\left(\beta_{j}<\beta^{(\ell)}\right)$ and $r_{j}^{(\kappa)}<$ $R^{(\ell)}<R^{(u)}<R_{j}\left(\rho_{\min }\right)<r_{j}^{\left(\kappa^{\prime}\right)}$ [similar to statement (i)]; the system does not have a feasible solution, as well. Otherwise, we define the admissible region

$$
E 3=\left\{\begin{array}{l}
R_{j}: R_{j} \in \mathfrak{R}, \forall \kappa \in \mathbf{I}_{r}, r_{j}^{(\kappa)} \notin \mathfrak{R}, \\
\max \left(R^{(\ell)}, R_{j}\left(\rho_{\min }\right)\right) \leq R_{j} \leq R^{(u)}
\end{array}\right\} .
$$

This implies that if $R_{j}^{*} \in E 3, R_{j}^{*}=R^{(u)}$.

As an example, consider a three-class system. Assume that the users are distributed among the classes as follows: $40 \%$ in class- $0,30 \%$ in each class- 1 and class- 2 . The $P G$ is $G=61$. Finally, we select $\gamma_{n}=15 \mathrm{~dB}$ common to all classes. The QoS requirements are assumed to be $\beta_{0}=4 \mathrm{~dB}, \beta_{1}=8 \mathrm{~dB}$, and $\beta_{2}=12 \mathrm{~dB}$ for class- 0 , class- 1 and class- 2 , respectively. Fig. 2 depicts the rate-characteristic polynomial by which the
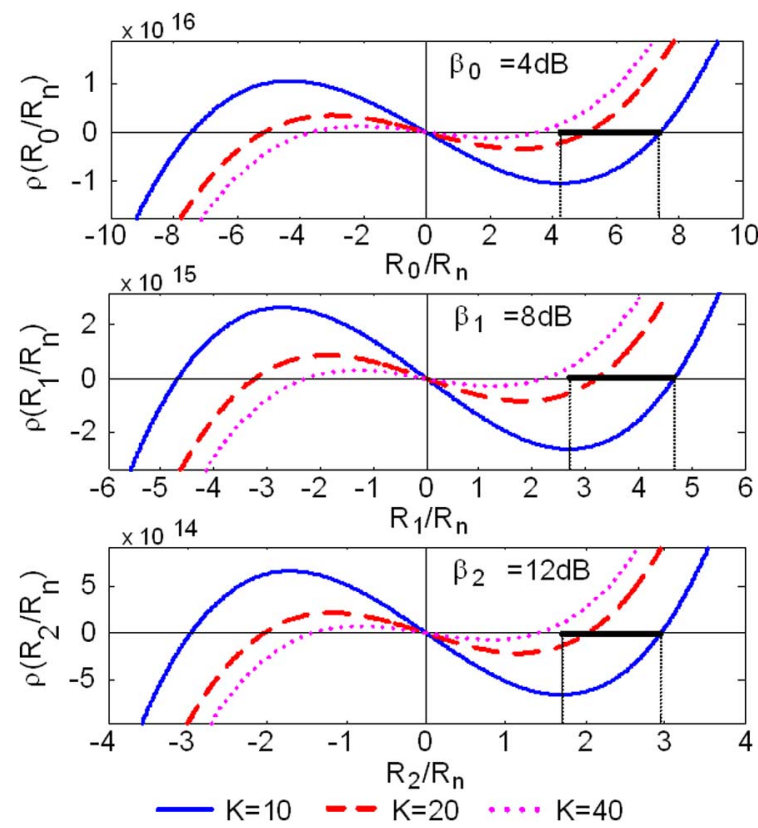

Fig. 2. The rate characteristic polynomials of three classes for $L=1024$, $\alpha=1.5$, and $\gamma_{n}=15 \mathrm{~dB}$.

transmission-rate feasible regions for the three classes are determined, given that the nominal packet time-slot is $L=1024$ bit-period and assuming $\alpha=1.5$. For instance, for $K=10$ users, the admissible regions $E 1 \cup E 2$ (in boldface line) of class- 0 , class- 1 and class- 2 are $\left\{4.25 \leq R_{0} / R_{n} \leq 7.41\right\} \cup \emptyset$, $\left\{2.71 \leq R_{1} / R_{n} \leq 4.67\right\} \cup \emptyset$, and $\left\{1.72 \leq R_{2} / R_{n} \leq 2.95\right\} \cup \emptyset$, respectively. Clearly, when $K$ and $\beta_{j}$ exceed certain values, there will be no feasible solution. Thus, the numerical results in Fig. 2 absolutely comply with the theoretical results obtained in Lemma 2 and Lemma 3.

\section{B. Power Allocation and Control Scheme}

The optimal transmission power for every class of users is obtained throughout the following lemma.

Lemma 4: The optimal transmission power of users in any class-j, transmitting at the maximum admissible rate $R_{j}^{*}$ is given by

$P_{j}^{*}= \begin{cases}\pi_{j}=\alpha \frac{\beta_{j}}{\gamma_{n}} & \text { for } \beta^{(\ell)} \leq \beta_{j} \leq \beta^{(u)} \\ \frac{\frac{\beta_{j}}{1+\frac{\beta_{j}}{\gamma^{(\ell)}}}}{\gamma_{n}\left(\sum_{s=0}^{S-1} \frac{K_{s}}{\left.1+\frac{\beta_{s}}{\gamma^{(\ell)}}-(K-1)\right)}<\pi_{j}\right.} & \text { for } \beta_{j}<\beta^{(\ell) .} .\end{cases}$

and $P_{j}^{*}$ is not feasible for $\beta_{j}>\beta^{(u)}$.

$$
\begin{aligned}
& E 1=\left\{\begin{array}{l}
R_{j}: R_{j} \in \mathfrak{R}, \exists \kappa \in \mathbf{I}_{r}, \\
\max \left(R^{(\ell)}, R_{j}\left(\rho_{\min }\right)\right) \leq R_{j} \leq r_{j}^{(\kappa)} \leq \min \left(R_{j}\left(\rho_{\max }\right), R^{(u)}\right)
\end{array}\right\} \\
& E 2=\left\{\begin{array}{l}
R_{j}: R_{j} \in \mathfrak{R}, \exists \kappa \in \mathbf{I}_{r}, \\
\max \left(R^{(\ell)}, R_{j}\left(\rho_{\max }\right)\right) \leq R_{j}=r_{j}^{(\kappa)} \leq \min \left(R_{j}\left(\rho_{\min }\right), R^{(u)}\right)
\end{array}\right\} .
\end{aligned}
$$


Proof: From Lemma 1 and Lemma 3, we have that for $\beta^{(\ell)} \leq \beta_{j} \leq \beta^{(u)}$ the maximum admissible transmission rate of any class- $j$ is achieved when $\gamma_{j}=\gamma_{j}^{*}$. Substituting this in (18), we get $P_{j}^{*}=\pi_{j}, \forall j$. On the other hand, for $\beta_{j}<\beta^{(\ell)}$, we have from Lemma $3 R_{j}^{*}=R^{(u)}$. This means that lowering the QoS below $\beta^{(\ell)}$ does not yield a rate increment above the upper-bound cutoff-rate $R^{(u)}$. This yields $\bar{\sigma}_{I_{k_{j}}}^{2}\left(R_{j}^{*}\right)=\bar{\sigma}_{\max }^{2}$ and, hence, $\gamma_{j}=\gamma^{(\ell)}$. Substituting the last term in (18) we get $P_{j}^{*}<\pi_{j}$. Also, for $\beta_{j}>\beta^{(u)}$ there is no feasible transmission rate and thus, no feasible power.

Observation: When $\beta_{j}<\beta^{(\ell)}$, the resource allocation is uncontrollable. It results directly from Lemma 3 and Lemma 4 that both $R_{j}^{*}$ and $P_{j}^{*}$ are independent of the control parameter $\alpha$. Therefore, both resources are uncontrollable.

In light of the above observation, we adopt $\beta^{(\ell)} \leq \beta_{j} \leq \beta^{(u)}$ as the QoS operating interval for the remaining analysis.

\section{CAPACITY ANALYSIS}

In most network applications, researchers attempt to raise the amount of the transmitted bits while keeping network power consumption as low as possible [6]. Therefore, it is logical to model the capacity in our network as the ratio of the total transmission rate to the total transmission power which is given by

$$
C=\frac{\sum_{j} p^{(j)} R_{j}^{*}(\alpha)}{\sum_{j} p^{(j)} P_{j}^{*}(\alpha)}=\frac{\sum_{j} K_{j} R_{j}^{*}(\alpha)}{\sum_{j} K_{j} P_{j}^{*}(\alpha)}
$$

where $R_{j}^{*}(\alpha)$ is the transmission rate for class- $j$ users given in (41) and obtained by Lemma 3 . On the other hand, $P_{j}^{*}(\alpha)$ is the laser transmission power for class- $j$ users given in (43) and obtained by Lemma 4 . If we consider $R_{j}^{*}$ normalized by $R_{n}$, then $C$ can be seen as the per-user network capacity normalized by $R_{n}$ per unit of power. Our proposed criterion consists of finding the optimal control parameter $\alpha^{*}$ that maximizes $C(\alpha)$ such as

$$
\alpha^{*}=\arg \max _{\alpha>1}\{C(\alpha)\} .
$$

As a result, $P_{j}^{*}\left(\alpha^{*}\right)$ and $R_{j}^{*}\left(\alpha^{*}\right)$ are optimally controlled via $\alpha^{*}$.

Furthermore, we define the nominal capacity when all users transmit at nominal rate $R_{n}$ as

$$
C_{n}=\frac{R_{n}}{\sum_{j} p^{(j)} P_{j}^{*}}=\frac{R_{n}}{\alpha \sum_{j} p^{(j)} \beta_{j}} \gamma_{n} .
$$

The control parameter is obtained as $\alpha_{n}^{*}=$ $\arg \max _{\alpha>1}\left\{C_{n}(\alpha)\right\}$. This implies $\alpha_{n}^{*}=\min (\alpha>1)$.

\section{NumericAl ReSUlTS AND DisCUSSION}

In this section, we provide a numerical analysis of our analytical results in order to gain more insight into the problem. In

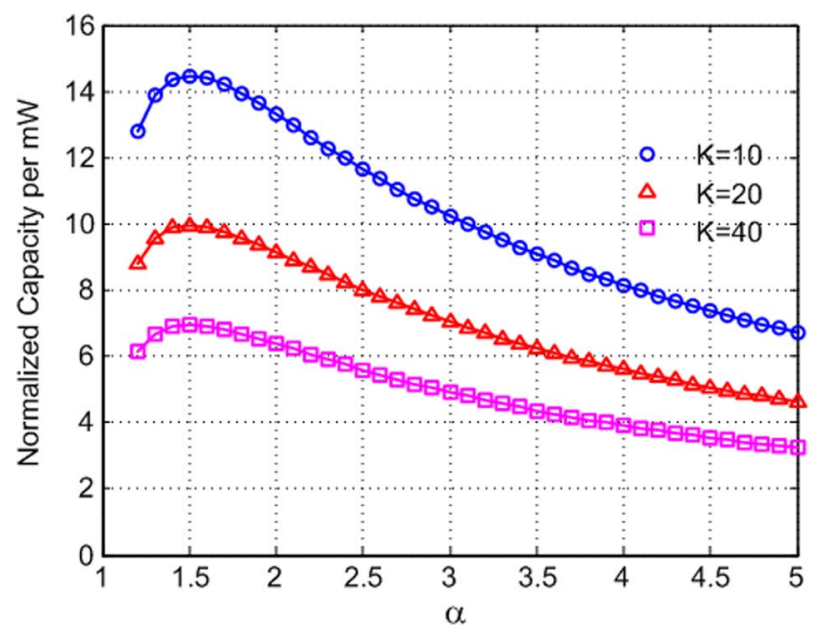

Fig. 3. The network capacity shape versus the control parameter $\alpha$.

addition, the analytical results are then compared to numerical ones obtained using a numerical search method. This method is the BFGS (suggested by Broyden, Fletcher, Goldfarb, and Shannon in 1970) [16]. It finds numerically the optimal transmission rate and the optimal transmission power of class $-j$ users that maximize $C(\alpha)$ as functions of $\alpha$ such that $\alpha>1$. The optimization problem is subject to the constraints $S I R_{j}=\beta_{j}$, $R^{(\ell)} \leq R_{j} \leq R^{(u)}$ and $0<P_{j} \leq \pi_{j}$. The numerical results show a total agreement with the analytical ones and validate our analysis.

We consider a three-class system, $S=3$, where each class is characterized by its own QoS. The total number of users in the network changes from $K=10$ to 40 users. The users are distributed among the classes as follows: $40 \%$ in class- $0,30 \%$ in class- 1 , and $30 \%$ in class- 2 . All the users are assigned codes of fixed PG $G=61$ through which they can generate packets of length $L=1024$ bits per time slot. Finally, we select $\gamma_{n}=$ $15 \mathrm{~dB}$ common to all classes. Assume that QoS requirements are $\beta_{0}=4 \mathrm{~dB}, \beta_{1}=8 \mathrm{~dB}$, and $\beta_{2}=12 \mathrm{~dB}$ for class- 0 , class- 1 and class-2, respectively.

The normalized network capacity is examined in Fig. 3 as a function of the control parameter $\alpha$. Notice that the normalized capacity per user has a global maximum, which means that there exists a value of $\alpha$ that maximizes the normalized capacity. Obviously, in this case it is 1.5 . As $\alpha$ goes to infinity, the user's capacity degrades. Consequently, the high optical intensity-level turns out to be destructive, which fits to our target revealed in the model of the capacity function depicted in (44).

Fig. 4 illustrates the optimal capacity $C$ versus the nominal capacity $C_{n}$ per active user when the number of users increases. The figure shows clearly the improvement of the optimized capacity per user with respect to the nominal one which can be considered as a lower bound. A large capacity is provided in the region of small number of users because more bits can be tolerated per time slot due to overlapping. As $K$ increases, this capacity gets reduced until it eventually reaches the nominal one.

The optimal transmission power of each class of users as a function of $K$ is shown in Fig. 5. We observe that the allocated transmission power per user is fairly chosen for each class according to its QoS requirement. Class-2 users transmit with the 


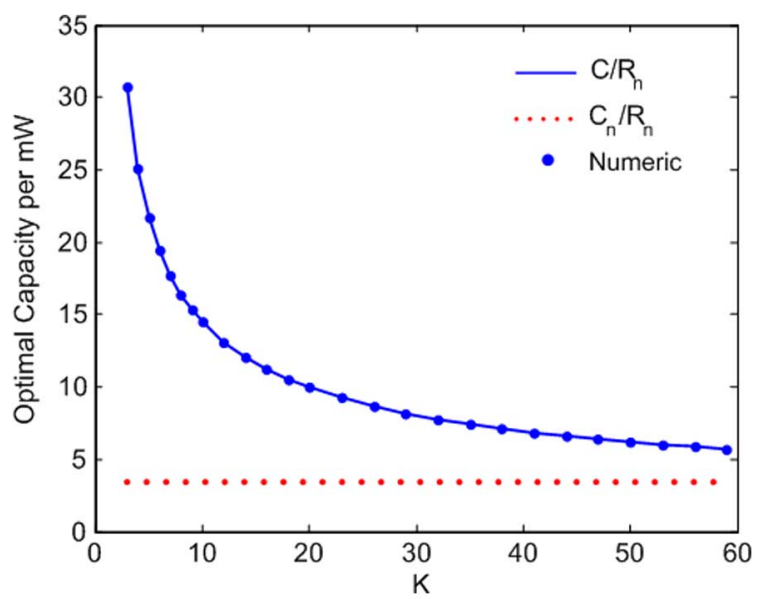

Fig. 4. The improved and the nominal capacities, normalized to $R_{n}$.

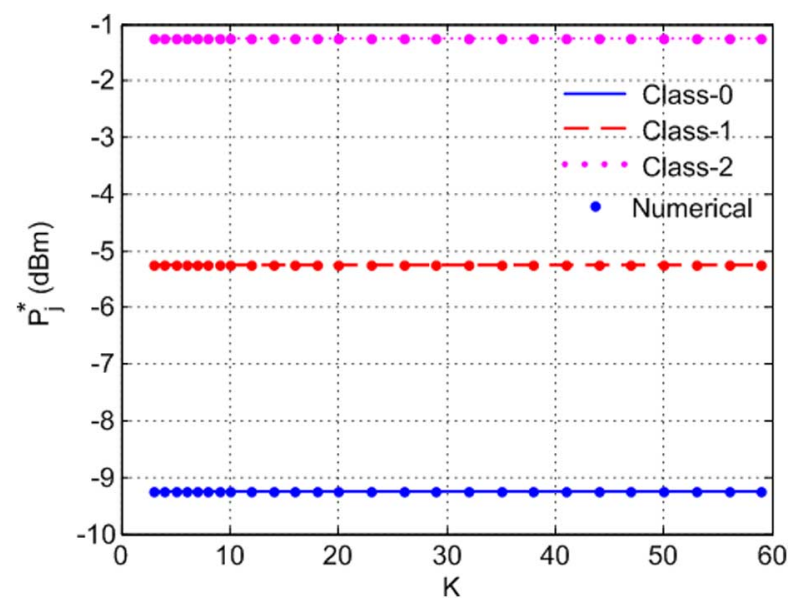

Fig. 5. The optimal transmission power versus the total number of users.

highest power because they require the highest QoS. In addition, users always transmit with the highest power $\pi_{j}$, a result which validates Lemma 4.

The optimal transmission rates for each class of users are depicted in Fig. 6. Using our proposed algorithm, it is clear that there is an increase in the transmission rate of each class of users. All classes can simultaneously transmit at rates varying between the lower and the upper bound cutoff rates of the OV-CDMA system. Note that in [7], only one class at a time can transmit at rate between the cutoff bounds while the other classes transmit either at the lower or at the upper cutoff bound, a fact which can be considered as unfair. As $K$ increases, each user is required to diminish the transmission bit rate to reduce the MAI effect in the optical channel.

In the remaining part, we evaluate the effect of the QoS on the proposed resource allocation and control algorithm. In this direction, we keep the same QoSs of class-0 and class- 2 as in the previous part and we vary that of class- 1 . The other design parameters remain unchanged.

The proposed controlling scheme adjusts the transmission rate of class- 1 as $\beta_{1}$ varies to fit the requirements as illustrated in Fig. 7, while $R_{0}^{*}$ and $R_{2}^{*}$ are constant. The highest transmission rate is allocated when a poor QoS is required. Then, whether

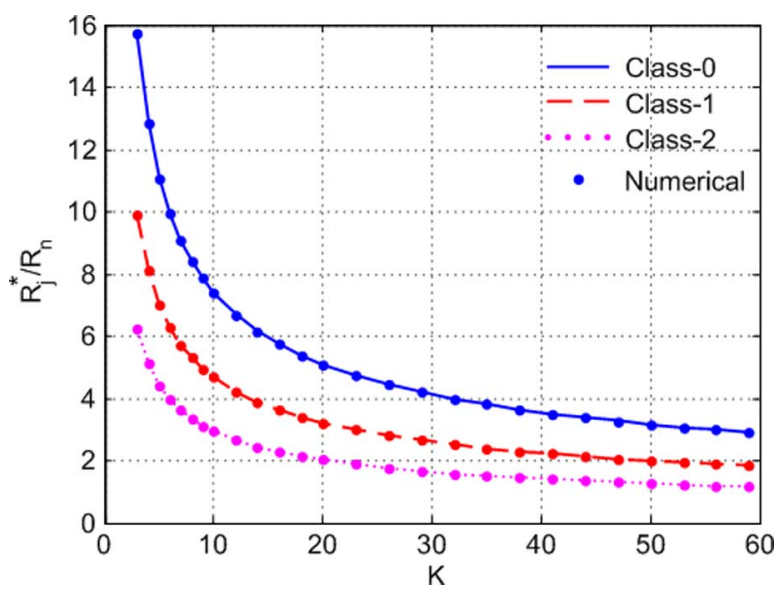

Fig. 6. The normalized optimal transmission rate of class- $j$ users versus the total number of users.

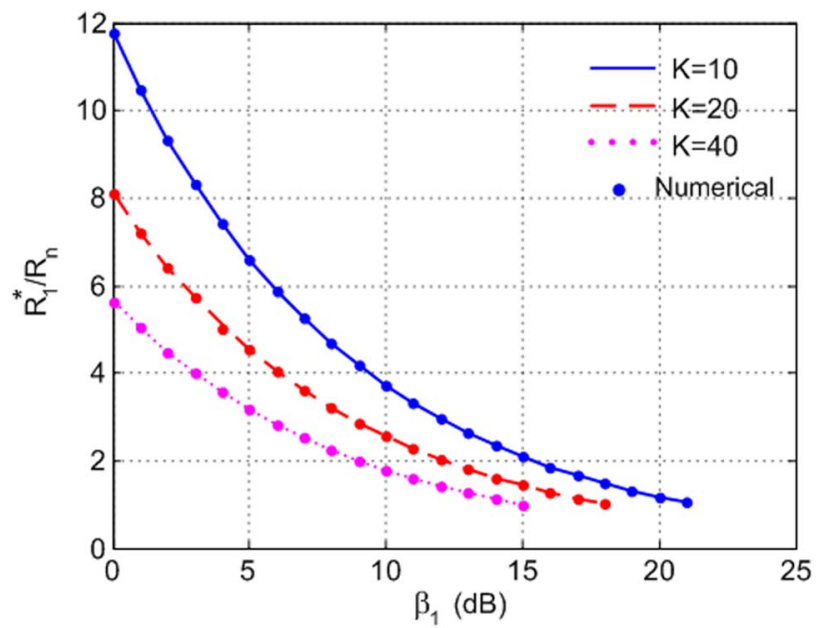

Fig. 7. The normalized optimal transmission rate of class-1 users versus QoS.

more users become active or a stringent QoS is demanded, $R_{1}^{*}$ decreases toward the nominal rate. When $\beta_{1}>\beta^{(u)}$, we remark that high QoSs are no more tolerable since they force the transmission rate to go below the nominal rate. This in turn validates the analytical result presented in Lemma 3.

The power control versus the rate allocation of class- 1 is revealed in Fig. 8. Low rate users are illuminated with high power since they transmit at a high QoS. As the allotted transmission rate increases, the corresponding supplied power decays rapidly because the QoS has been relaxed. Moreover, when $K$ increases, less power is allowed per user and the maximum allowable increase in the transmission rate above the nominal rate is smaller.

The normalized optimal network capacity per user is depicted in Fig. 9. The improvement of the capacity with respect to the nominal capacity is obvious for different network conditions. For example, in the presence of 20 active users six of which are in class- 1 transmitting at a target $\operatorname{QoS} \beta_{1}=0 \mathrm{~dB}$, the maximum achievable capacity is 17.7 times $R_{n}$ per $\mathrm{mW}$, which is around 4 times the nominal capacity. Such an improvement diminishes when the number of active users increases, and/or when the demand on QoS increases. The fast capacity decay is due to the 


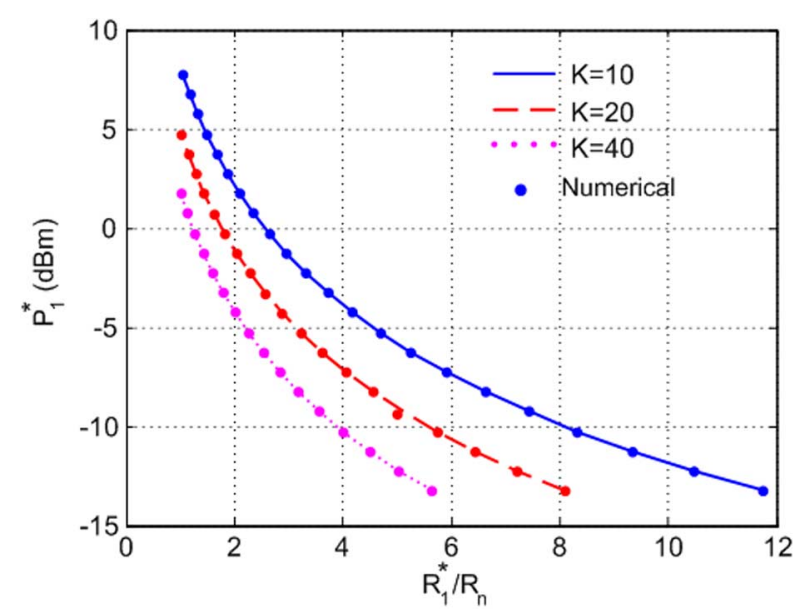

Fig. 8. The optimal transmission power allocation as a function of the normalized optimal transmission rate of class-1 users.

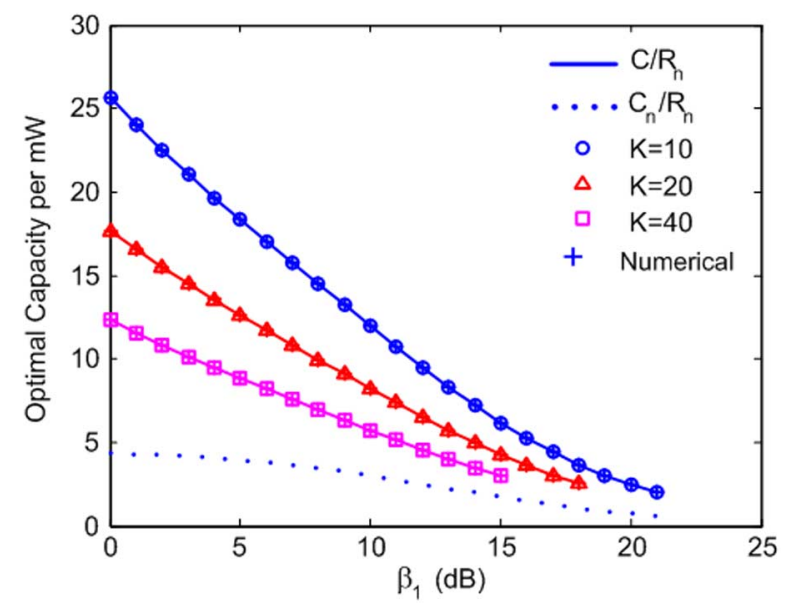

Fig. 9. The normalized optimal user's capacity versus the QoS variation of class-1.

fact that the controlling scheme tends to guarantee high QoS requirements rather than to increase the transmission rate of users by reducing the MAI effect in the network.

\section{CONCLUSION}

We proposed a fair resource allocation scheme for multiclass time-slotted optical OV-CDMA-based packet networks. This scheme is fair as it is based on the QoS requirements of the users. In addition, no class of users can dominate the other classes. The Sherman-Morrison's matrix inversion lemma was used to solve the power allocation problem in a closed form. A rate-characteristic polynomial has been derived and solved for the optimal transmission rate allocation. The resources are optimally allocated as functions of a single control parameter, which helps to reduce the search space into one dimensional space. Our approach is unified since it is independent from the shape of the network capacity. Both numerical and analytical results proved that our scheme is simple to implement and offers a substantial improvement to the system capacity.

\section{APPENDIX}

Polynomial coefficients in Section III

$$
\begin{aligned}
A_{4}= & G L^{4}-12 G L^{3}+51 G L^{2}-90 G L+54 G \\
A_{3}= & 5 G L^{4}-(42 G+6 F) L^{3}+(102 G+30 F) L^{2} \\
& -(54 G+48 F) L-27 G+24 F \\
A_{2}= & 8 G L^{4}-(34 G+6 F) L^{3}-(G-6 F) L^{2} \\
& +(60 G+24 F) L-9 G-24 F \\
A_{1}= & 4 G L^{4}+(8 G+6 F) L^{3}-(40 G+30 F) L^{2} \\
& +(12 G+24 F) L \\
A_{0}= & (8 G+6 F) L^{3}-(4 G+6 F) L^{2} \\
B_{3}= & G L^{2}-6 G L+9 G \\
B_{2}= & 3 G L^{2}-(6 G-2 F) L-9 G-2 F \\
B_{1}= & -4 F L^{2}+(12 G+4 F) L \\
B_{0}= & 2 F L^{3}-(4 G+2 F) L^{2} .
\end{aligned}
$$

\section{REFERENCES}

[1] L. G. Kazovsky, W. Shaw, D. Gutierrez, N. Cheng, and S. Wong, "Nextgeneration Optical access networks," J. Lightw. Technol., vol. 25, no. 11, pp. 3426-3444, Nov. 2007.

[2] E. Inaty, H. M. H. Shalaby, and P. Fortier, "On the cutoff rate of a multiclass OFFH-CDMA system," IEEE Trans. Commun., vol. 53, no. 2, pp. 323-334, Feb. 2005

[3] C. W. Sung and W. S. Wong, "Power control and rate management for wireless multimedia CDMA systems," IEEE Trans. Commun., vol. 49, no. 7, pp. 1215-1226, Jul. 2001.

[4] A. Kwasinski and N. Farvardin, "Resource allocation for CDMA networks based on real-time source rate adaptation," in Proc. IEEE ICC $C N F$, May 2003, vol. 5, pp. 3307-3311.

[5] S. Oh, D. Zhang, and K. M. Wasserman, "Optimal resource allocation in multiservice CDMA networks," IEEE Trans. Wireless Commun., vol. 2, no. 4, pp. 811-821, Jul. 2003.

[6] D. Zhang, S. Oh, and N. T. Sindhushayana, "Optimal resource allocation for data service in CDMA reversed link," IEEE Trans. Wireless Commun., vol. 6, no. 10, pp. 3648-3656, Oct. 2007.

[7] R. Raad, E. Inaty, P. Fortier, and H. M. H. Shalaby, "Optimal resource allocation scheme in a multirate overlapped optical CDMA system," $J$. Lightw. Technol., vol. 25, no. 8, pp. 2044-2053, Aug. 2007.

[8] R. Raad, E. Inaty, P. Fortier, and H. M. H. Shalaby, "Optical S-ALOHA/CDMA system for multirate applications: architecture, performance evaluation, and system stability," J. Lightw. Technol., vol. 24, no. 5, pp. 1968-1977, May 2006.

[9] E. Inaty, H. M. H. Shalaby, P. Fortier, and L. A. Rusch, "Multirate optical fast frequency hopping CDMA system using power control," $J$. Lightw. Technol., vol. 20, no. 2, pp. 166-177, Feb. 2002.

[10] H. Yashima and T. Kobayashi, "Optical CDMA with time hopping and power control for multirate networks," J. Lightw. Technol., vol. 21, no. 3, pp. 695-702, Mar. 2003.

[11] T. Miyazawa and I. Sasase, "Multirate and multiquality transmission scheme using adaptive overlapping pulse-position modulator and power controller in optical network," in Proc. IEEE ICON, Nov. 2004, vol. 1, pp. 127-131.

[12] N. G. Tarhuni, M. S. Elmusrati, T. O. Korhonen, and E. Mutafungwa, "Multiaccess-interference mitigation using power control in optical-CDMA star networks," in Proc. IEEE ICC, May 2005, vol. 3, pp. 1593-1597.

[13] T. M. Bazan, D. Harle, and I. Andonovic, "Mitigaton of beat noise in time-wavelength opitcal code-division multiple-access systems," $J$. Lightw. Technol., vol. 24, no. 11, pp. 4215-4222, Nov. 2006.

[14] G. Keiser, Optical Fiber Communication, 3rd ed. New York: McGraw-Hill, 2000.

[15] J. Sherman and W. J. Morrison, "Adjustment of an inverse matrix corresponding to a change in one element of a given matrix," Ann. Math. Statist., vol. 21, no. 1, pp. 124-127, 1950.

[16] L. Cooper, Applied Nonlinear Programming for Engineers and Scientists. Englewood, NJ: Aloray, 1974. 


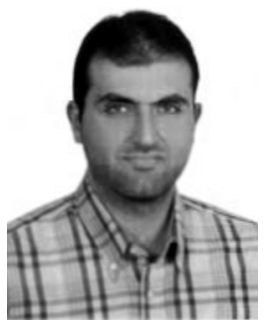

Elie Inaty (S'99-M'02) was born in El-Koura, North Lebanon, on June 23, 1975. He received the B.S. and M.S. degrees in electrical engineering from the University of Balamand, Lebanon, in 1996 and 1998, respectively. He received the Ph.D. degree from the Université Laval, Canada, in 2001.

He is now an Assistant Professor at the University of Balamand and Adjunct Professor with the Université Laval. His research interests include CDMA and WDM fiber optic communications, network control and resource management issues in optical communication networks, and radio multiple-access techniques.

Robert Raad was born in Rahbé, North Lebanon, on August 24, 1978. He received the B.S. and M.S. degrees in computer engineering from the University of Balamand, El-Koura, North Lebanon.

Currently, he is working toward the Ph.D. degree at the Université Laval, Canada. His research interests include optical CDMA, network-control, and resource management issues in optical communications networks.

Mr. Raad had received an award from the professors of faculty of engineering at the University of Balamand in 2003.

Paul Fortier (SM'00) received the B.Sc. and M.Sc. degrees in electrical engineering from Laval University, Québec, Canada, in 1982 and 1984, respectively, and the M.S. degree in statistics and the Ph.D. degree in electrical engineering from Stanford University, Stanford, CA, in 1987 and 1989, respectively.

Since 1989, he has been with the Department of Electrical and Computer Engineering, Laval University, where he is currently a full professor. From 1991 to 1997, he was Program Director for the B.Sc. degree in computer engineering and, from 1997 to 2003, he was Chairman of the Department of Electrical and Computer Engineering. Since 2003, he has been Associate Dean for Develop- ment and Research with the Faculty of Science and Engineering. He has done consulting work for several companies and government agencies in Canada. His research interests include digital signal processing for communications and the study of complexity and performance tradeoffs in hardware implementations, with applications in wireless and optical communications. He has been involved in the organization of national and international conferences and workshops in these fields.

Dr. Fortier is a Fellow of the Engineering Institute of Canada.

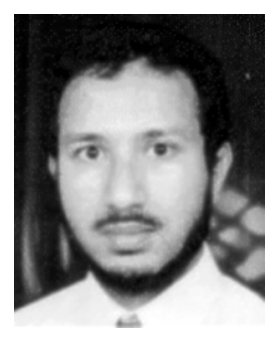

Hossam M. H. Shalaby (S'83-M'91-SM'99) was born in Giza, Egypt, in 1961. He received the B.S and M.S. degrees from the University of Alexandria, Egypt, in 1983 and 1986, respectively, and the Ph.D. degree from the University of Maryland, College Park, in 1991, all in electrical engineering.

In 1991, he joined the Department of Electrical Engineering, University of Alexandria, Egypt, as an Assistant Professor. He was promoted to an Associate Professor in 1996 and to Professor in 2001, which is his current position. Since December 2000, he has been an Adjunct Professor with the Department of Electrical and Information Engineering, Faculty of Sciences and Engineering, Laval University, Quebec, Canada. From March to April 1996, he was a Visiting Professor with the Electrical Engineering Department, Beirut Arab University, Lebanon. From September 1996 to January 1998, he was an Associate Professor with the Electrical and Computer Engineering Department, International Islamic University Malaysia, and from February 1998 to December 1998, he was with the School of Electrical and Electronic Engineering, Nanyang Technological University, Singapore, where he was a Senior Lecturer. From January 1999 to February 2001, he was an Associate Professor. His research interests include optical communications, optical CDMA, spread-spectrum communications, and information theory. 